\title{
Bi-objective Optimization of Logistic Networks with Full-Connectivity Structure Using NSGA-II
}

\author{
Przemysław Ignaciuk and Łukasz Wieczorek
}

\begin{abstract}
This paper addresses the resource control issue in goods distribution networks. Two types of actors-suppliers and warehouses - are linked without topological restrictions. The interconnection structure in the discussed class of logistic networks forms a mesh-type topology. During the distribution process, the warehouses face external demands, not known $a$ priori. The flow of goods in the system is governed according to the networked order-up-to (NOUT) inventory management policy implemented in a centralized manner. The balance between the customer service level and the holding cost poses a bi-objective optimization challenge. The novelty of this paper is an application of the nondominated sorting genetic algorithm II to adjust the NOUT policy to the logistic problem under consideration. Numerical studies performed for various topologies and distribution structures have asserted the efficiency of the discussed method.
\end{abstract}

Index Terms-Logistic networks, genetic algorithms, multi-objective optimization, uncertain demand, inventory management.

\section{INTRODUCTION}

\section{A. Literature Review}

In recent times, the growth of global trade and continuous economic development have broadened the scale of enterprise operations. This process occurs in numerous economy branches, and one branch where it is highly observable is logistics. Today, supply chains are becoming more and more extensive and complex. In addition, there are a number of factors that affect the efficiency of the distribution process independent of the actual industry under consideration: for example, uncertain demand [1], [2], international sanctions [3], [4], or changing climate [5], [6]. The efficiency of the inventory control process is an important factor for the proper operation of companies in a variety of contexts such as food [7], healthcare [8], or defense [9]. The real-life distribution environments are affected by several uncertain factors. One of the most common challenges is customer demand with the evolution not known a priori, which is taken explicitly into account in this work. Another frequent challenge arises from lead-time delays occurring in communication channels, which leads to the necessity of early planning as well as caching all the orders in-transit. This issue is especially damaging in the case of perishable resource distribution. References [10], [11] analyze distribution systems with deteriorating resources and

Manuscript received January 5, 2019; revised April 12, 2019.

The authors are with the Institute of Information Technology, Lodz University of Technology, 90-924 Łódź, Poland (e-mail: przemyslaw.ignaciuk@p.lodz.pl, lukasz.wieczorek.1@edu.p.lodz.pl). propose an inventory control policy that counteracts the negative influence of time perturbations.

To date, literature related to the optimization of supply chains has mostly been focused on systems with significant topological constraints such as the following:

- single-stage systems [12], [13],

- multistage, serial organizations [14], [15], and

- hybrid star-bus structures [10], [16].

However, in practical realization, these topologies are being replaced by more complex structures having no connectivity restrictions. The multi-objective optimization (MO) problem of resource control in such mesh-type topologies is difficult to solve; for example, it is not amenable to the standard convex analysis and related numerical solution methods, or probability theory (e.g., Markov chains). The first major challenge is to form a mathematical model of such a complex topology that is suitable for efficient numerical treatment. Next, one needs to define objective functions that will allow one to perform simulation-based optimization with well-defined criteria.

Reference [17] presents a comprehensive review of scientific literature related to MO issues in supply chain management (SCM) using evolutionary algorithms (EAs) based on articles published in 2005-2015. The trend of an increasing amount of research in this subject is noticeable, especially in recent years. Most of the considered publications are related to the fields of industrial and manufacturing engineering as well as computer science. The authors discuss in detail the application of several types of MO methods for various types of logistic networks. They refer to supply chains with diverse mathematical models, connectivity structures, and optimization objectives, except for cost reduction (which was a common objective for all the analyzed articles). The review conclusions encourage one to develop, apply, and investigate various MO techniques for optimization issues of logistic networks.

Another literature analysis performed in 2016 [18] indicates genetic algorithms (GAs) as one of the most popular methods of optimizing sophisticated real-life problems. That paper provides a comprehensive review of the literature positions, which have been published since the late 1990s. According to the Council of Supply Chain Management Professionals, the authors divide analyzed papers into three processes of SCM:

- Manufacturing flow management-42\% participation.

- Order fulfillment-41\% participation.

- Demand management-7\% participation.

The rest of the research under consideration-about $10 \%$ - is related to other aspects (e.g., returns management, supplier relationship management, and product development 
and commercialization). The authors appreciate the different methods of application of GA-based optimization in SCM despite the development of several other soft computing techniques. However, they note a trend of formulating MO hybrid methods based on classical GA implementations in order to optimize modern logistic networks.

A recent paper from the adduced literature reviews [19] indicates that the distribution uncertainties considered in this work coincide with the current research directions of SCM. Today, customer demands are difficult to predict and are not known a priori, which is a challenge in operations planning and control processes and ought to be investigated in more detail. This means that instead of deterministic demands, stochastic demands should be applied to the distribution processes under consideration. Moreover, the authors emphasize the importance of MO optimization in inventory control issues. Hybrid solutions based on GAs have been suggested as a potential subject of further studies.

\section{B. Problem Statement}

This work considers the organization of the goods distribution process in mesh-type logistic networks having arbitrary connectivity structure. The analyzed topologies do not assume any simplifications or restrictions in the connection structure, except for the case of isolated (without any incoming or outgoing interconnections) and self-replenishing nodes. The efficiency assessment of the distribution process is performed using two criteria:

- customer service level (SL) and

- overall holding cost (HC).

The first criterion quantifies customer satisfaction. It is measured as a percentage of fulfilled external demand from all orders imposed on the warehouses. The objective is to maximize the SL value. The second criterion is to minimize economic costs, quantified through HC (i.e., the total cost of maintaining stocks in the logistic system). In practice, the second criterion implies the reduction of excess stock in the warehouses.

The contribution of this paper to network management is to apply the nondominated sorting genetic algorithm II (NSGA-II) to optimize the goods distribution process in mesh-type logistic systems. The application of NSGA-II allows one to tune the operation of networked order-up-to (NOUT) inventory management policy in the considered class of distribution systems. The resulting Pareto-optimal set allows network managers to flexibly adjust the policy parameters to given environmental conditions and performance objectives.

\section{LOGISTIC SYSTEM}

\section{A. Distribution Process}

The considered class of distribution networks comprises two types of actors: controlled nodes (warehouses) and external sources (suppliers). The goal of the resource distribution process is to enable the controlled nodes to satisfy external demands. Initially, each controlled node stores a nonzero stock. The external sources provide goods to the network to replenish the stock depleted according to the demands. All the nodes are able to store a limited amount of resources in their warehouses. The nodes are linked with each other using directed interconnections, forming a mesh topology. The connectivity structure assumes that no node can be separated (i.e., located outside the network) or replenish itself. Resource distribution is realized to maintain inventory balance in the network (i.e., to provide replenishment for the controlled nodes affected by demands). The flow of goods proceeds along interconnections described by a pair of parameters, typical for the logistic problem under consideration:

- supplier contribution (SC) - the part of required resources that a node requests from a specific supplier and

- lead-time delay (LTD) - the time, including both shipment preparation and transport from a supplier to the destination node, associated with the replenishment order.

The sequence of operations performed at a controlled node in any period of time is presented in Fig. 1. First, each controlled node registers resources from incoming shipments into the stock. Then, it strives to satisfy imposed external demands. Finally, all the nodes attempt to maintain the inventory network balance by fulfilling replenishment signals originating from nearby warehouses.

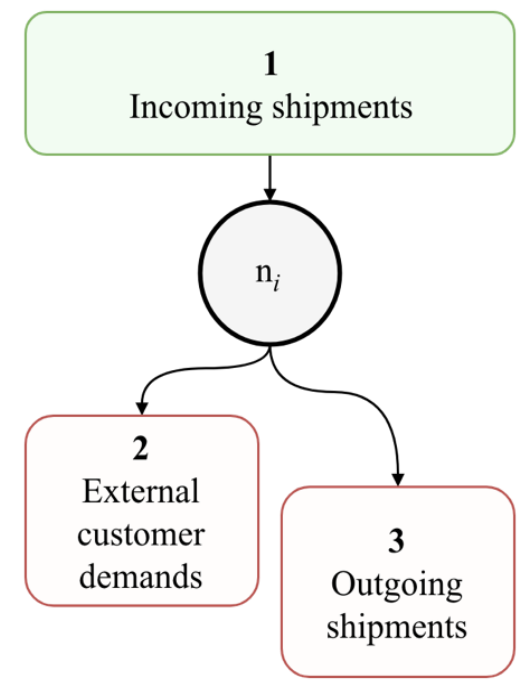

Fig. 1. Node procedural sequence.

\section{B. Analytical Background}

The logistic system under consideration is composed of $N$ controlled nodes and $M$ external sources indexed through $\Phi_{N}=\{1,2, \ldots, N\}$ and $\Phi_{M}=\{1,2, \ldots, M\}$, respectively. In addition, let $\Phi_{i}$ indicate a set of all the suppliers linked to a particular node $i$. Each interconnection between two nodes $i$ and $j$ is described by a pair of attributes $\left(\alpha_{i j}, \beta_{i j}\right)$. The first one (i.e., $\alpha_{i j}$ ) denotes the SC between nodes, $\alpha_{\mathrm{ij}} \in[0,1]$. The sum of the SCs from all the suppliers connected to a particular controlled node equals 1 . The second attribute is the LTD between these two nodes. It comprises shipment preparation and transportation time $\beta_{i j}=\beta_{i}^{\text {prep }}+\beta_{i j}^{\text {trans }}, \beta_{i j} \in[1, B]$, where $B$ denotes the highest LTD between any linked node. For convenience, the basic symbols used in the analysis of the mathematical network model have been grouped in Table I. Moreover, Fig. 2 illustrates the model of the analyzed logistic 
system based on the procedural sequence depicted in Fig. 1.

Similarly, the quantity of goods sent by node $i$ to the other

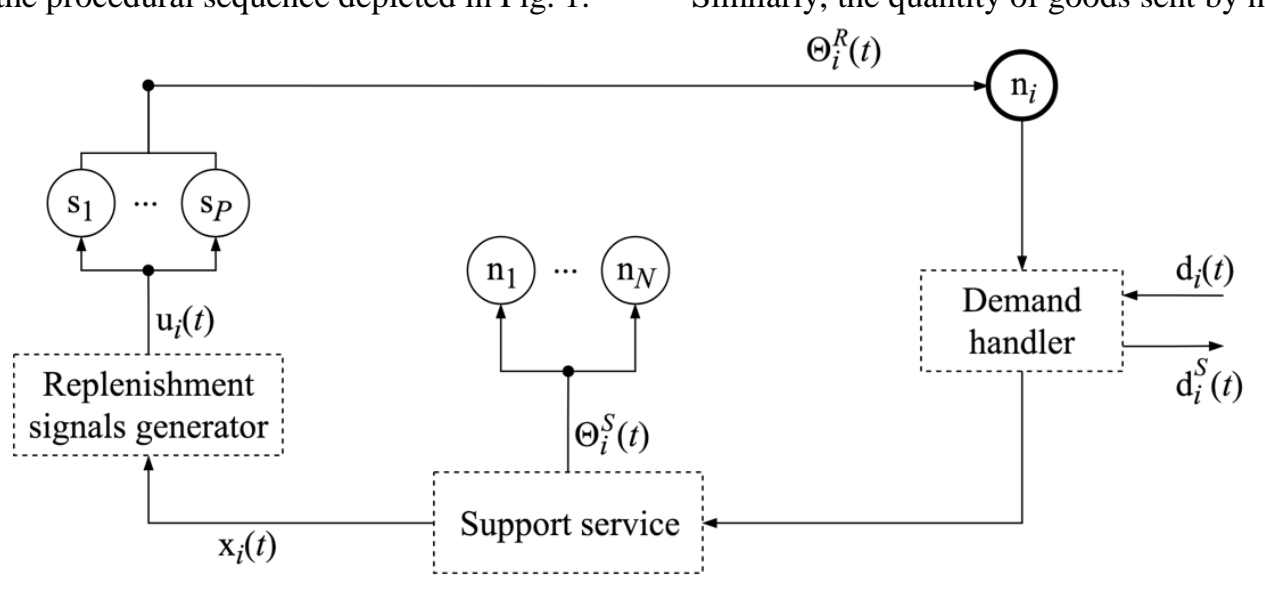

Fig. 2. Inventory management system.

\section{TABLE I: MATHEMATICAL NOTATION}

\begin{tabular}{|c|c|}
\hline Symbol & Meaning \\
\hline$t, k$ & $\begin{array}{l}\text { Period of time, where } t, k=1,2, \ldots, T \text { for the distribution } \\
\text { process horizon } T\end{array}$ \\
\hline$P=N+M$ & Total number of nodes in the network \\
\hline$x_{i}(t)$ & On-hand stock level at node $i$ in period $t$ \\
\hline$d_{i}(t)$ & External demands imposed on node $i$ in period $t$ \\
\hline$d_{i}^{S}(t)$ & External demands fulfilled by node $i$ in period $t$ \\
\hline$u_{i}(t)$ & Replenishment signals generated by node $i$ in period $t$ \\
\hline$\Theta_{i}^{R}(t)$ & $\begin{array}{l}\text { Quantity of goods from the replenishment orders } \\
\text { received by node } i \text { in period } t\end{array}$ \\
\hline$\Theta_{i}^{S}(t)$ & $\begin{array}{l}\text { Quantity of goods in the replenishment orders sent by } \\
\text { node } i \text { in period } t\end{array}$ \\
\hline $\mathbf{x}(t)$ & Vector containing on-hand stock levels \\
\hline $\mathbf{u}(t)$ & Vector containing replenishment orders \\
\hline $\mathbf{d}(t)$ & Vector of imposed demands \\
\hline $\mathbf{d}^{\mathbf{S}}(t)$ & Vector of satisfied demands \\
\hline
\end{tabular}

Because of demand uncertainty, the controlled nodes may not always be able to satisfy the imposed demand in full. The quantity of satisfied demand is specified through time-varying functions,

$$
0 \leq d_{i}^{S}(t) \leq d_{i}(t)
$$

where $d_{i}^{S}(t)=d_{i}(t)$ means the case of full customer satisfaction.

On the basis of preliminary theoretical considerations [20], [21], the stock balance at controlled node $i$ at time $t$ may be described as

$$
x_{i}(t+1)=x_{i}(t)+\Theta_{i}^{R}(t)-d_{i}^{S}(t)-\Theta_{i}^{S}(t)
$$

The quantity of goods in the replenishment orders received by node $i$ is equal to the sum of all orders sent by its suppliers (both controlled nodes and external sources) and may be calculated as

$$
\Theta_{i}^{R}(t)=\sum_{j=1}^{P} \alpha_{j i} u\left(t-\beta_{j i}\right) .
$$

controlled nodes in response to the replenishment signals equals

$$
\Theta_{i}^{S}(t)=\sum_{j=1}^{N} \alpha_{i j} u_{j}\left(t-\beta_{i}^{\text {prep }}\right) .
$$

According to (1) and the assumed sequence of operations performed by a controlled node (Fig. 1), the quantity of demands satisfied by node $i$ in period $t$ is determined as

$$
d_{i}^{S}(t)=\min \left\{x_{i}(t)+\Theta_{i}^{R}(t), d_{i}(t)\right\}
$$

\section{State-Space Representation}

For efficient implementation, the proposed mathematical model is represented in a matrix-vector form. Let us define a matrix $\Psi_{k}$, where $k \in\{1,2, \ldots, N\}$, that stores information about the network structure (i.e., the associations between nodes) as

$$
\Psi_{k}=\left[\begin{array}{cccc}
\sum_{i: B_{i 1}=k} \varphi_{i 1} & \omega_{12} & \cdots & \omega_{1 N} \\
\omega_{21} & \sum_{i: B_{i 2}=k} \varphi_{i 2} & \cdots & \omega_{2 N} \\
\vdots & \vdots & \ddots & \vdots \\
\omega_{N 1} & \omega_{N 2} & \cdots & \sum_{i: B_{i N}=k} \varphi_{i N}
\end{array}\right]
$$

where we have the following:

- entries on the main diagonal $\sum_{i: \mathrm{B}_{i j}=k} \varphi_{i j}$ represent incoming shipments according to the orders placed at $\mathrm{LTD}=k$ periods before and

- off-diagonal entries $\omega_{i j}$,

$$
\omega_{i k}=\left\{\begin{array}{c}
-\varphi_{i k}, \text { if } \beta_{i}^{\text {prep }}=k \\
0 \text { otherwise. }
\end{array}\right.
$$

Additionally, let us introduce a summary matrix $\Psi$,

$$
\Psi=\sum_{k=1}^{B} \Psi_{k}
$$


According to the notation presented in Table I and the node interconnection structure specified by (6), the dynamic state-space model may be described as

$$
\mathbf{x}(t+1)=\mathbf{x}(t)+\sum_{k=1}^{B} \Psi_{k} \mathbf{u}(t-k)-\mathbf{d}^{\mathbf{s}}(t) .
$$

\section{D.NOUT Inventory Policy}

The management of the goods distribution process in the logistic system under consideration is realized using the NOUT policy. This strategy was proposed in [21], based on the classical OUT policy adjusted for networked structures. Once the inventory-related operations visualized in Fig. 1 are completed, each controlled node generates a replenishment signal for its suppliers. Owing to the uncertainty of future demands, the manager of a controlled node does not know the exact amount of resources to be ordered. The NOUT policy needs to define a target stock level (TSL) for each controlled node. This value is a reference level that the node strives to achieve in each period of time. Let us define a vector containing the TSLs of all the controlled nodes as

$$
\mathbf{x}^{\mathbf{T}}=\left[x_{1}^{\mathrm{T}}, x_{2}^{\mathrm{T}}, \ldots, x_{N}^{\mathrm{T}}\right]
$$

The operation of generating replenishment signals for the suppliers assumes obtaining information about the on-hand stock level as well as the goods in-transit in the shipments issued by suppliers. The quantity of resources that should be ordered by the controlled nodes from their suppliers may be calculated from

$$
\mathbf{u}(t)=\Psi^{-1}\left[\mathbf{x}^{\mathbf{T}}-\mathbf{x}(t)-\sum_{k=1}^{B} \sum_{\tau=k}^{B} \Psi_{k} \mathbf{u}(t-\tau)\right]
$$

The adjustment of the NOUT policy for a particular distribution network consists in proper selection of the TSL vector. The optimal solution needs to achieve a high SL while reducing excess goods in stock. According to [2], if a fixed external demand equal to the highest value is imposed on each controlled node, the TSL vector that provides satisfying customer demands in full may be determined from

$$
\mathbf{x}^{\mathbf{T}}=\left(\mathbf{I}_{N}+\sum_{k=1}^{B} k \Psi_{k}\right) \Psi^{-1} \mathbf{d}_{\max }
$$

However, this vector is only a reference solution for the multicriteria optimization process, because its application results in a significant amount of excess resources in the warehouses (and thus a high $\mathrm{HC}$ generated by the network) when the demands exhibit temporal variations.

\section{BI-OBJECTIVE OPTIMIZATION}

The bi-objective optimization problem considered in this work aims to provide a set of potential solutions (TSL vectors) without having to apply a full-search approach for reduced computational time. The number of candidate solutions for the analyzed class of systems is significant and numerically prohibitive for larger constructs. For this reason, EAs are being applied in logistics in recent studies. A continuous genetic algorithm (CGA) has been proposed to find a desired TSL vector based on predefined optimization priorities [22]. In this work, NSGA-II is used so that one does not need to define optimization directions in advance. The algorithm returns a set of "good" solutions, from which the network manager may choose an optimal one for a given situation and network topology.

Let us define two objective functions that should be minimized as follows:

- $f_{1}$ - the function grouping information about the quantity of goods stored in controlled nodes,

$$
f_{1}=\sum_{t=0}^{T} \mathbf{x}(t)
$$

- $f_{2}$ - the function of unsatisfied demands (the percentage of all the demands imposed on the network that have not been satisfied),

$$
f_{2}=\sum_{t=0}^{T} \mathbf{d}^{\mathrm{s}}(t) / \sum_{t=0}^{T} \mathbf{d}(t)
$$

The implemented algorithm initially generates a random population of candidate solutions based on a reference TSL vector calculated using (12). Fig. 3 displays an example population in relation to the defined objective functions. Then, for each individual in the population, a simulation of the distribution process is performed to determine the objective function values. The next step is to determine, for each candidate solution, the fitness values and crowding distance (i.e., the distance from the nearest other individuals). Later, the typical operations for GAs (i.e., selection, crossover, and mutation) are performed. NSGA-II has been implemented according to the assumptions listed in [23].

In Fig. 3, the Pareto set- the set of best solutions in a given population - is marked by red dots.

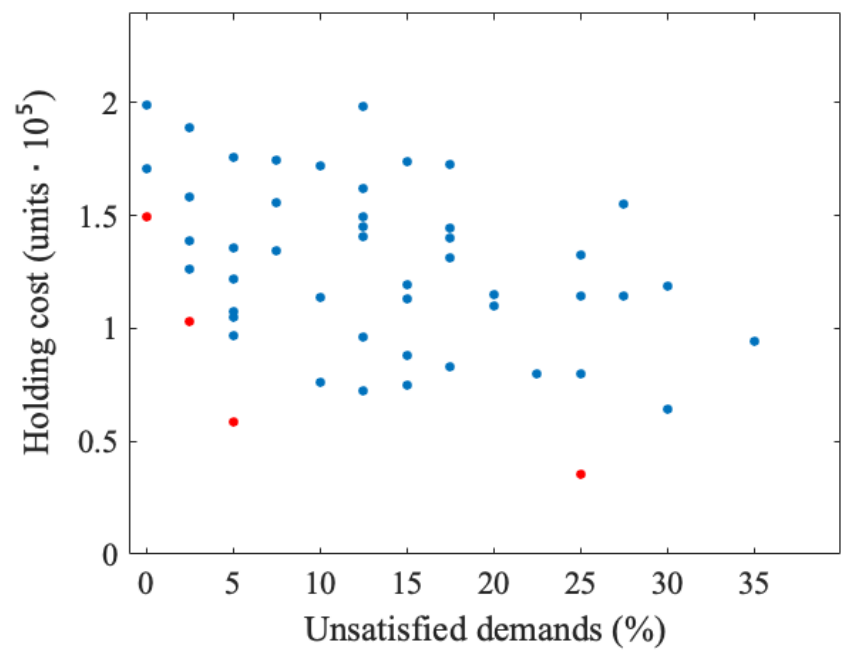

Fig. 3. Result visualization for a random population. 


\section{NUMERICAL STUDY}

The effectiveness assessment of the application of NSGA-II in the considered class of problems required one to perform numerous simulations. The simulations were carried out about $10^{4}$ times for different topologies and system parameters (i.e., network size, interconnection structure, and connection complexity). The example results are presented for the topology from Fig. 4. The structure encompasses 15 controlled nodes and five external sources. The distribution process lasts for 30 periods, and LTDs are set in the range [1], [5]. The external demand was generated using stochastic Gamma distribution with scale and shape parameters set as 5 and 10, respectively. The implemented NSGA-II assumes multipoint crossover and candidate tournament selection. The population consists of 50 candidate solutions, the mutation probability equals $30 \%$, and the stop condition is set as $10^{4}$ generations.

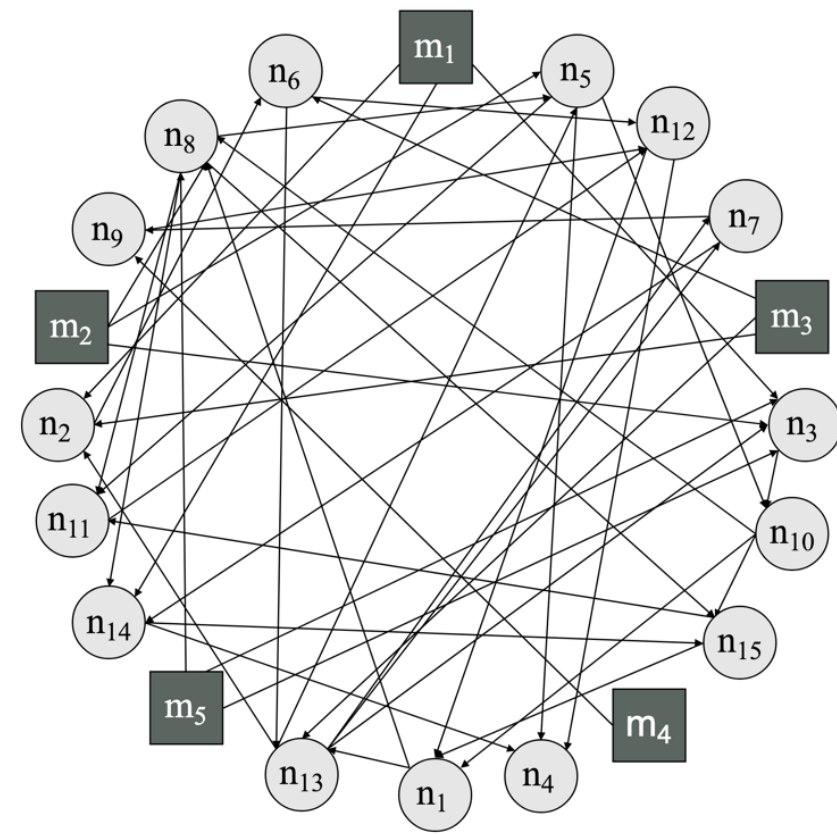

Fig. 4. Network topology.

The initial TSL vector calculated using (12) equals

$$
\mathbf{x}^{\mathbf{T}}=\left[\begin{array}{c}
2078,771,704,317,2875,589,537,2415 \\
967,1588,900,854,1310,851,1876
\end{array}\right]
$$

On the basis of this vector, a full-search algorithm would have to perform $1.94 \cdot 10^{45}$ simulations of the resource distribution process, whereas NSGA-II needed only $0.5 \cdot 10^{6}$ to search the given space of solutions. Fig. 5 illustrates the obtained results from the NSGA-II optimization process. The blue dots represent outcomes of bi-objective optimization performed using individuals (TSL vectors) from the considered populations of the algorithm. The red dots highlight the Pareto set: that is, the set of most desirable solutions for a system manager to choose from for an intended SL and HC according to given environmental conditions. Table II details the candidate solutions from the Pareto set, and Appendix A contains all the corresponding TSL vectors.

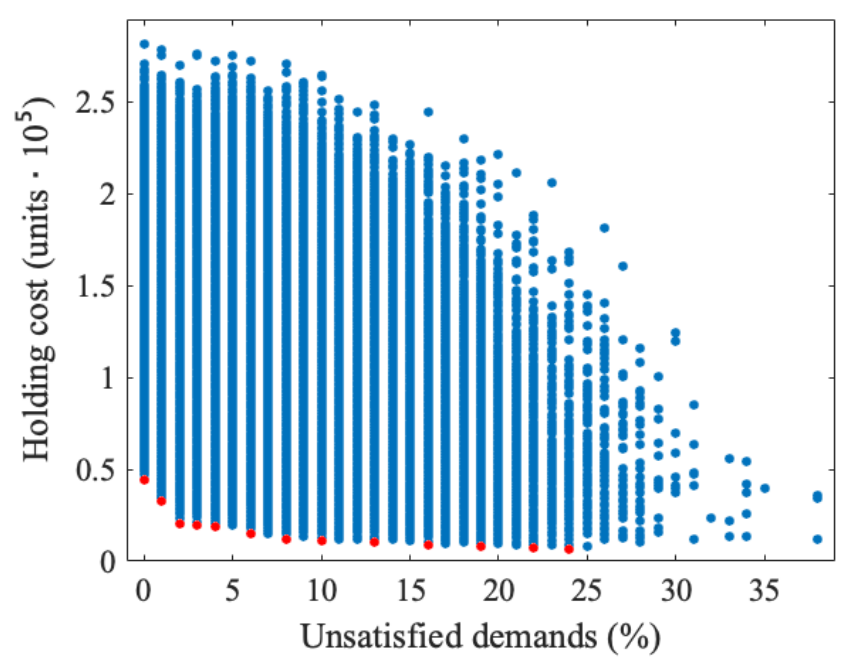

Fig. 5. Result visualization for a random population.

The optimization process does not assume any more operations. It is a question of a system manager to select which of the proposed solutions allows one to maintain the best trade-off between the optimization objectives: that is, to best fulfill the environmental requirements. Depending on the acceptable level of unsatisfied demands, the $\mathrm{HC}$ may be reduced significantly. For instance, an acceptance of sacrificing $10 \%$ of customer demands leads to reducing the $\mathrm{HC}$ four times.

TABLE II: NSGA-II OPTIMIZATION RESULTS: PARETO SET

\begin{tabular}{lll}
\hline \hline Reference & Unsatisfied demand $(\%)$ & Holding cost (units $\left.\cdot 10^{5}\right)$ \\
\hline A1 & 0 & 0.44551 \\
A2 & 1 & 0.32518 \\
A3 & 2 & 0.20491 \\
A4 & 3 & 0.19995 \\
A5 & 4 & 0.18751 \\
A6 & 6 & 0.14795 \\
A7 & 8 & 0.11834 \\
A8 & 10 & 0.11080 \\
A9 & 13 & 0.10745 \\
A10 & 16 & 0.08612 \\
A11 & 19 & 0.08075 \\
A12 & 22 & 0.07603 \\
A13 & 24 & 0.06452 \\
\hline \hline
\end{tabular}

For comparison, let us adduce the optimization of a similar logistic problem using the CGA described in [22]. In that method, evolution proceeds on the basis of a fitness function combining all the objectives together. The optimization process focuses mainly on the solution search space that best satisfies the predefined fitness function. Therefore, the optimization process needs to be performed several times with different parameters to obtain a similar set of results as in the case of NSGA-II. In contrast to the CGA with a single criterion, bi-objective optimization focuses on solutions that are close to both intended objectives - $\mathrm{HC}$ reduction and high SL guarantee-achieved simultaneously with less computational effort. 


\section{CONCLUSIONS}

This paper investigates the application of NSGA-II in the optimization of resource distribution in mesh-type logistic networks. The goods flow control is realized using a networked inventory policy implemented in a centralized manner. The considered logistic system model includes uncertain demands imposed on the network. The resource distribution takes into account two optimization goals: (i) to provide a high customer SL defined as the proportion of fulfilled demands to all the imposed requests and (ii) to reduce excessive goods storage in the node warehouses so as to minimize HC. The application of NSGA-II allows one to significantly decrease the solution search space and to obtain the desired collection faster than when using the classical full-search approach. In further research, additional aspects typical for the analyzed class of systems may be considered through flexible model extension (e.g., back-order costs).

\section{APPENDIX A}

Equations (A1)-(A13) are TSL vectors of Pareto-optimal solutions obtained in the NSGA-II optimization process discussed in Section IV. All the listed solutions are equally good: that is, they cannot be objectively compared.

$$
\begin{aligned}
& \mathbf{x}_{\mathrm{P} 1}^{\mathbf{T}}=\left[\begin{array}{c}
801,752,508,300,725,7,344,718, \\
521,498,194,742,682,474,976
\end{array}\right] ; \\
& \mathbf{x}_{\mathrm{P} 2}^{\mathbf{T}}=\left[\begin{array}{c}
772,392,331,293,1269,84,208,931, \\
424,501,355,328,802,387,758
\end{array}\right] ; \\
& \mathbf{x}_{\mathrm{P} 3}^{\mathbf{T}}=\left[\begin{array}{c}
665,388,333,312,461,291,147,214, \\
375,495,623,215,346,558,828
\end{array}\right] ; \\
& \mathbf{x}_{\mathrm{P} 4}^{\mathbf{T}}=\left[\begin{array}{c}
542,459,425,297,709,79,141,301, \\
411,260,289,332,723,255,948
\end{array}\right] ; \\
& \mathbf{x}_{\mathrm{P} 5}^{\mathbf{T}}= {\left[\begin{array}{c}
844,432,136,277,1082,145,271,671, \\
354,230,328,329,433,377,378
\end{array}\right] ; } \\
& \mathbf{x}_{\mathrm{P} 10}^{\mathbf{T}}=\left[\begin{array}{c}
72,319,196,310,680,134,149,46, \\
156,180,545,138,120,288,474
\end{array}\right] ; \\
& \mathbf{x}_{\mathrm{P} 8}^{\mathbf{T}}=\left[\begin{array}{c}
643,364,247,134,687,117,127,411, \\
304,36,204,439,650,243,392
\end{array}\right] ; \\
& \mathbf{x}_{\mathrm{P} 7}^{\mathbf{T}}=\left[\begin{array}{c}
509,317,285,194,539,118,160,571, \\
189,220,236,679,367,196,677
\end{array}\right] ;
\end{aligned}
$$

$$
\begin{gathered}
\mathbf{x}_{\mathrm{P} 11}^{\mathbf{T}}=\left[\begin{array}{c}
455,160,99,25,358,67,99,488, \\
153,375,112,515,268,436,665
\end{array}\right] ; \\
\mathbf{x}_{\mathrm{P} 12}^{\mathrm{T}}=\left[\begin{array}{c}
118,324,165,79,308,247,101,96, \\
236,379,33,364,461,125,443
\end{array}\right] ; \\
\mathbf{x}_{\mathrm{P} 13}^{\mathrm{T}}=\left[\begin{array}{c}
608,294,245,45,21,26,184,336, \\
200,238,639,361,11,181,66
\end{array}\right] .
\end{gathered}
$$

\section{ACKNOWLEDGMENT}

This work was performed within the framework of the research project titled "Genetic algorithms in the optimization of logistic networks" founded by the Institute of Information Technology, Lodz University of Technology.

\section{REFERENCES}

[1] T. Xiao and D. Yang, "Price and service competition of supply chains with risk-averse retailers under demand uncertainty," Int. J. Prod. Econ., vol. 144, pp. 187-200, July 2008.

[2] P. Ignaciuk and $Ł$. Wieczorek, "Optimization of mesh-type logistic networks for achieving max service rate under order-up-to inventory policy," Adv. Intell. Syst., vol. 657, pp. 118-127, Sept. 2017.

[3] C. Harlanda, R. Brenchleyb, and H. Walkera, "Risk in supply networks," J. Purch. Supply Manag., vol. 9, pp. 51-62, Mar. 2003.

[4] H. Davarzani, R. Z. Farahani, and H. Rahmandad, "Understanding econo-political risks: impact of sanctions on an automotive supply chain," Int. J. Oper. Prod. Man., vol. 35, pp. 1567-1591, Jan. 2015.

[5] F. Y. Chen and C. A. Yano, "Improving supply chain performance and managing risk under weather-related demand uncertainty," Manage. Sci., vol. 56, pp. 1380-1397, Jun. 2010.

[6] Q. Miao, M. K. Feeney, F. Zhang, E. W. Welch, and P. S. Sriraj, "Through the storm: Transit agency management in response to climate change," Transport. Res. D-Tr. E., vol. 63, pp. 421-432, Aug. 2018.

[7] P. J. Wu and P. C. Huang, "Business analytics for systematically investigating sustainable food supply chains," J. Clean. Prod., vol. 203, pp. 968-976, Dec. 2018.

[8] I. W. G. Kwon, S. H. Kim, and D. G. Martin, "Healthcare supply chain management; strategic areas for quality and financial improvement," Technol. Forecast. Soc., vol. 113, pp. 422-428, Dec. 2016.

[9] E. Gholz, A. D. James, and T. H. Speller, "The second face of systems integration: An empirical analysis of supply chains to complex product systems," Res. Policy, vol. 47, pp. 1478-1494, May 2018.

[10] P. Ignaciuk, "LQ optimal and robust control of perishable inventory systems with multiple supply options," IEEE T. Automat. Contr., vol. 58, pp. 2108-2113, Aug. 2013.

[11] P. Ignaciuk, "Discrete-time control of production-inventory systems with deteriorating stock and unreliable supplies," IEEE Trans. Syst. Man. Cybern. Syst., vol. 45, pp. 338-348, Aug. 2014.

[12] D. Castellano, E. Gebennini, A. Grassi, T. Murino, and B. Rimini, "Stochastic modeling of a single-vendor single-buyer supply chain with (s, S)-inventory policy," IFAC PapersOnLine, vol. 51, pp. 974-979, Sep. 2018.

[13] P. Ignaciuk and A. Bartoszewicz, "Dead-beat and reaching-law-based sliding-mode control of perishable inventory systems," B. Pol. Acad. Sci-Tech., vol. 59, pp. 39-49, Aug. 2011.

[14] N. Sbai and A. Berrado, "A literature review on multi-echelon inventory management: the case of pharmaceutical supply chain,' MATEC Web Conf., vol. 200, 13, Sep. 2018.

[15] K. Govindan and M. Fattahi, "Investigating risk and robustness measures for supply chain network design under demand uncertainty: A case study of glass supply chain," Int. J. Prod. Econ., vol. 183, pp. 680-699, Jan. 2017.

[16] P. Ignaciuk, "Nonlinear inventory control with discrete sliding modes in systems with uncertain delay," IEEE T. Ind. Inform., vol. 10, pp. 559-568, Jan. 2014

[17] T. Trisna, M. Marimin, Y. Arkeman, and T. C. Sunarti, "Multi-objective optimization for supply chain management problem: A literature review," Decis. Sci. Lett., vol. 5, pp. 283-316, Oct. 2015. 
[18] S. K. Jauhar and M. Pant, "Genetic algorithms in supply chain management: A critical analysis of the literature," Sadhana-Acad. P. Eng. S., vol. 41, pp. 993-1017, Sept. 2016.

[19] C. K. H. Lee, "A review of applications of genetic algorithms in operations management," Eng. Appl. Artif. Intel., vol. 76, pp. 1-12, Sept. 2018.

[20] S. Axsäter, Inventory Control, New York, Springer International Publishing, 2015.

[21] P. Ignaciuk, "Dynamic modeling and order-up-to inventory management in logistic networks with positive lead time," in Proc. the 2015 IEEE International Conference on Intelligent Computer Communication and Processing, Romania, 2015.

[22] Ł. Wieczorek and P. Ignaciuk, "Intelligent support for resource distribution in logistic networks using continuous-domain genetic algorithms," in Proc. the IEEE Second International Conference on Data Stream Mining \& Processing, Ukraine, 2018.

[23] D. Simon, Evolutionary Optimization Algorithms, Hoboken, John Wiley \& Sons, 2013.

Przemyslaw Ignaciuk was born in Lodz, Poland, in 1981. $\mathrm{He}$ received MSc and $\mathrm{PhD}$ degrees from Lodz University of Technology and a DSc degree in technical sciences granted by the Systems Research Institute of the Polish Academy of Sciences.

He worked as a business analyst and IT system architect.
Presently, he is an associate professor at the Institute of Information Technology, Lodz University of Technology. His current research focuses on robust control, congestion control, dynamical optimization, and logistic network management.

Prof. Ignaciuk is an IEEE Senior Member. He has participated in ten research projects, and in two as a project leader. Moreover, he is a recipient of several research scholarships and the author of over a hundred international scientific papers.

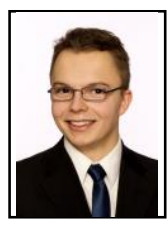

Lukasz Wieczorek received BSc and MSc degrees in computer science from Lodz University of Technology in 2015 and 2017, respectively. Presently, he is a PhD student at this university.

$\mathrm{He}$ is a software engineer with over five years of commercial experience. He is currently working in the Department of Autonomous Driving Maps at TomTom. His research areas are related to artificial intelligence, especially optimization issues in logistics. 